\title{
Lab safety matters
}

\author{
Jeffrey M Perkel \\ Overall safety is increasing, but new safety hazards, such as repetitive stress injuries and potential \\ toxicities from nanoparticles, are coming under closer scrutiny.
}

In the 1980s comedy Real Genius, laboratory safety is the stuff of sight gags and pratfalls. Playing the movie's protagonist, Val Kilmer protects himself from a sixmegawatt laser using Ray-Ban sunglasses and a baseball umpire's gear. Later, that laser's energy is harnessed to heat a housesized popcorn popper.

In reality, laser work is serious business, particularly for home-built systems arrayed on open optical benches, with the beam bouncing from mirror to mirror. Just ask Alipasha Vaziri, a research specialist at the Howard Hughes Medical Institute's Janelia Farm Research Campus. Vaziri builds laser-based systems for twophoton microscopy, a technique that uses high-peak-power, ultrafast pulsed lasers to excite the fluorophores in biological samples.

In addition to donning the usual safety eyewear found in every laser lab, Vaziri and his colleagues also remove watches, wedding rings and even hanging identification badges - anything that might possibly get into the beam path and reflect its light in an uncontrolled direction. He even avoids tying his shoes when the laser is on, so that his eyes will not cross the level of the laser.

To do their work effectively, multiphoton lasers must concentrate more power in short bursts (pulses). Although only a fraction of that power actually hits the sample, the laser systems typically deliver femtosecond pulses with peak pulse powers around 0.25 megawatts, Vaziri says, and members of his lab are developing a widefield multiphoton system using about 1,000 times higher pulse energies; by comparison, the typical one-photon microscopy diode laser puts out about 50 milliwatts of continuous power.

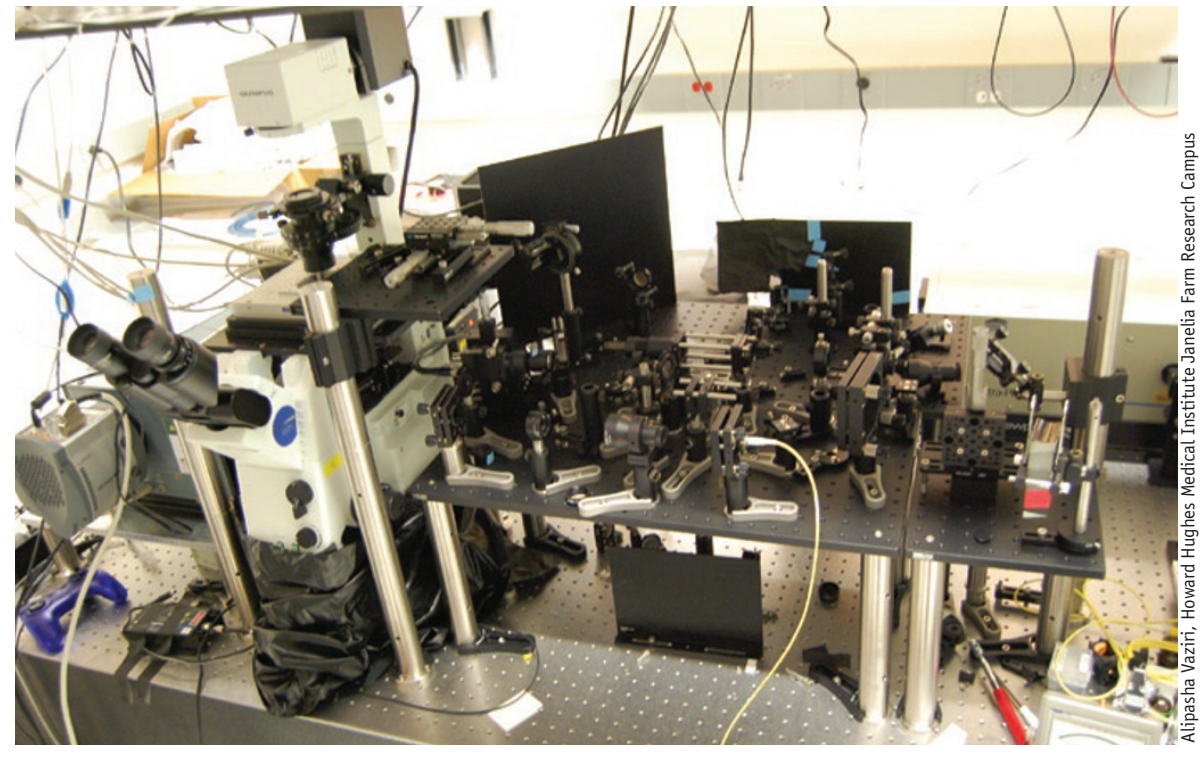

When testing new setups for multiphoton microscopy, researchers remove jewelry and avoid motions that could direct laser beams into their eyes.

For biological samples, multiphoton techniques are relatively gentle; long wavelengths, though undetectable to the human eye, penetrate deeper into the sample than visible light can and yet induce less phototoxicity. But for researchers' eyes, the lasers are anything but gentle. And their invisibility boosts the safety hazard, says Vaziri. "With visible lasers, your reflexes are fast and could mitigate some of the damage [that results from accidentally looking at the beam], but that is not true of invisible [infrared] lasers."

Such is life at the intersection of laboratory safety and technology development. As old techniques fall by the wayside, new technologies with new safety concerns arise to take their place. For the safety officers charged with protecting lab workers, the work environment is an ever-evolving challenge (Box 1).
New hazards, old hazards

Before embarking on their first experiments, new lab hires almost invariably undergo safety training in radiation, chemical and biological hazards. But there is only so much that such seminars can do. Researchers must actively consider how generic rules apply to their own workflows, says Susan Cook, a biological safety officer at Washington University in St. Louis. "It's incumbent on each lab [principal investigator] to build a culture of safety."

At the Wellcome Trust Sanger Institute near Cambridge, UK, researchers create risk assessments for every experiment (or class of experiment) they do, says Andrew White, campus health and safety manager.

A requirement of both UK and EU law, these five-page documents force researchers essentially to conduct a dry run of the 


\section{BOX 1 BIG TROUBLE IN LITTLE SCIENCE?}

Nano-sized particles often have radically different-and generally unknown-biological properties than their bulkier counterparts. As a general rule, however, they have the potential to cross biological barriers and induce both allergic and respiratory responses. Furthermore, the chemicals used to synthesize nanomaterials and structures, such as silane and hydrofluoric acid, are often acutely toxic.

According to Matt Finucane, executive director of environmental health and radiation safety at the University of Pennsylvania, those concerns were factored into the planning of a new

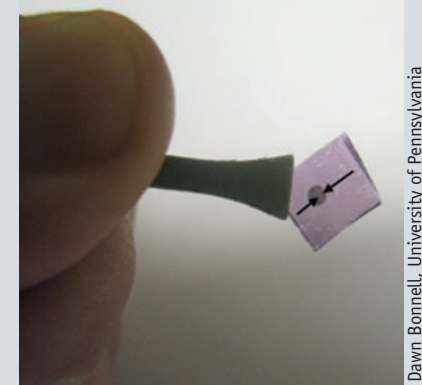

Nanoparticle fabrication brings new safety considerations. nanotechnology and microfabrication center on which the university expects to break ground this summer.

"This facility will have a lot of bells and whistles with respect to automatic shutoffs, alarm systems and so on, that are tiered to go off in response to different concentrations of materials in the air," Finucane says.

In most cases, however, general chemical hygiene strategies are sufficient to safely handle newly developed materials, says Dawn Bonnell, director of the Nano/ Bio Interface Center at the University of Pennsylvania. "[The chemical industry] has been inventing chemicals for a long time," she says. "There are standard protocols for what to do when developing things [that are] new and you don't know how they will behave."

Bonnell's team, which uses colloidal chemistry to pattern nanostructures, takes a twofold approach to nanoparticle safety: if at all possible, keep the materials suspended in liquid so researchers can neither inhale them nor release them into the air. If liquid handling is not possible, researchers should wear protective gear, including a respirator, eye wear, lab coat and gloves.

So far, so good: her team has not had an incident in 22 years of operation. But that does not mean they can rest on their laurels. Nanoscience, Bonnell notes, is heavily interdisciplinary; the possibility thus exists that a synthetic chemist who is well-versed in relevant lab safety practices will hand off some new nanomaterial to a biologist, say, who is not.

"We have to make sure there's cross-education about health and safety, and that's one of the things our center does," she says.

procedure while focusing on potential hazards and strategies to mitigate them. "It's about getting you to stop and think before you start work," explains White.

The safety assessment for SDS gel electrophoresis, for instance, lists among other things that powdered acrylamide should be avoided in favor of "proprietary ready-made solutions," and that 2-mercaptoethanol should be "used only in a fume cupboard."

For risk assessment to work, however, researchers must recognize the danger in the first place. Cook points out that even seemingly harmless flow cytometry poses a substantial biosafety threat if users are not properly trained. High-pressure flow lines can easily become clogged and cause cells to aerosolize, and researchers new to flow cytometry may not appreciate the risk, she says. That is where a solid rapport with lab safety personnel comes in handy, Cook says; "if you have a good relationship with people in the lab, they will come to us and say, 'I'm starting a new experiment, what do I need to know?"'

Her office advises most flow cytometry users to wear gloves, eye protection and a lab coat. If the researcher will be working with respiratory pathogens, such as influenza virus, she directs them to one of the university's two biosafety cabinetenclosed instruments, to protect against infectious aerosols.

Yet despite the best efforts of lab workers and safety officers, accidents will happen.

In early 2009, a 23-year-old laboratory worker at the University of California, Los Angeles, died of injuries sustained 
when a pyrophoric, or highly reactive, air-sensitive chemical, $t$-butyl lithium, ignited. As in many accidents, multiple things went wrong ${ }^{1}$. The syringe used for the synthesis, which was several inches shorter than recommended, malfunctioned, exposing the reactive liquid to the air. Unneeded hexane in the lab hood spilled and ignited as well. The researcher, Sheharbano Sangji, was not wearing a lab coat, and her clothes caught fire. A nearby safety shower went unused.

In labs and lab safety offices across the United States, the response was immediate introspection. At Yale University, Environmental Health and Safety director Peter Reinhardt and his staff undertook a census of campus labs to determine who was using such chemicals, how much they had on hand, and what they were using them for.

Of the 536 principal investigators that work with chemicals on campus, Reinhardt says, "we identified maybe 10 or 15 labs that have this chemical, of which maybe five use it on a regular basis."

For the most part, the census uncovered no problems; in those labs, researchers were using and storing the chemicals correctly, in accordance with the university's existing safety plan for pyrophoric chemicals. Yet members of his office also realized that the plan could use something of a makeover.

Yale University's previous plan, for instance, merely suggested a flameresistant lab coat and chemical-resistant gloves; the new policy requires them. "Before this incident, there were maybe a few people using flame-resistant lab coats, but not all," Reinhardt says. Under the new plan, "we made sure everyone had them," even purchasing protective equipment for researchers if needed.

Reinhardt and his colleague, Tom Ouimet, also developed a 14-minute online video demonstrating the proper techniques for working with these materials (http://www.yale.edu/ehs/onlinetraining/ OrganoLithium/OrganoLithium.htm).

The on-campus response, Reinhardt says, has been "excellent," and the video has become a popular resource in the lab safety training circuit nationwide, as well. Researchers, he notes, can view these webinars at their leisure or when they need a quick refresher on a particular technique, and can skip forwards and backwards through the material as needed. In addition, these videos provide a training mechanism
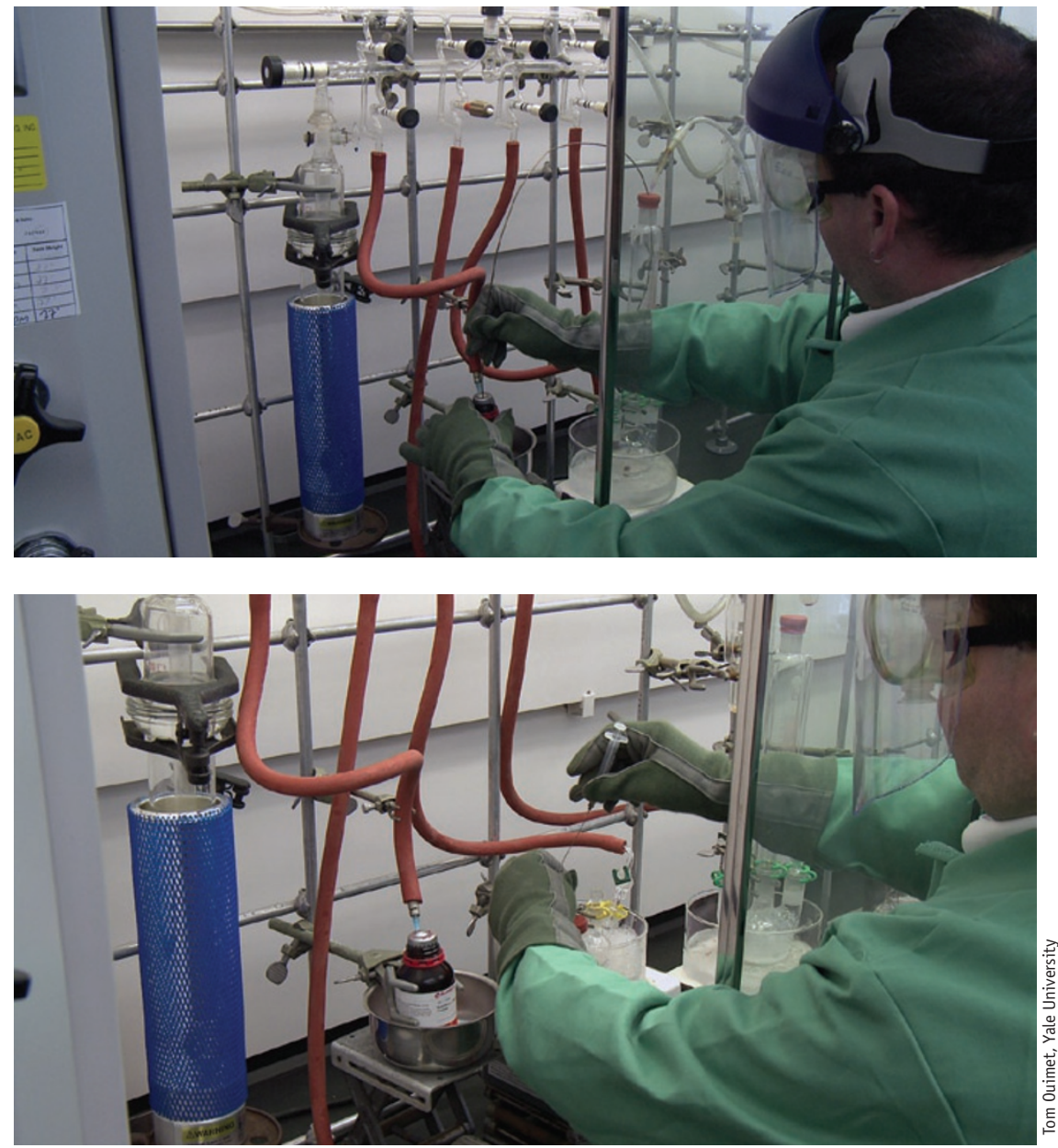

Researchers transfer an organolithium compound from a reagent bottle to a graduated funnel with a cannula (top) and to a reaction vessel with a syringe (bottom), avoiding exposing the chemical to air.

that can accommodate the rapid personnel turnover typical of academic labs.

Making training more accessible is one of the best things a department like his can do, says Reinhardt, whose office has produced dozens of training videos over the past decade. "Training is prevention, so we spend a lot of resources on it."

\section{Safety in numbers}

For the most part, such prevention works pretty well, and serious laboratory incidents are rare. According to the US Bureau of Labor Statistics, the rate of recordable incidents in scientific research and development services has steadily declined from 2.1 per 100 full-time employees in 2003 to 1.2 in 2008 (the most recent data available).

Mirroring that, Science Applications International Corporation (SAIC)Frederick, home to some 2,400 staff, has more than halved its accident rate among lab workers over the past decade, from 5.20 per 100 full-time employees in 2000 to 2.28 in 2009-lower even than among nonlaboratory administrative employees, according to occupational safety manager, Scott Keimig. "In effect, our laboratories are our safest work environment," Keimig says.

These numbers, of course, mostly reflect acute accidents; chronic injuries and exposure hazards are much more difficult to track. Declining accident rates can, in part, be attributed to researchers' increasing awareness of the dangers associated with the compounds and materials with which they work. Yet technical progress helps, too. DNA sequencing, for instance, used to require radioactive phosphorus, liters of electrophoresis running buffer, cumbersome gel rigs run at high voltage and large glass plates. Today, almost nobody sequences that way anymore, instead relying on fluorescent dyes, automated instruments and core facilities.

Automation and miniaturization tend to shrink or eliminate chemical exposure 

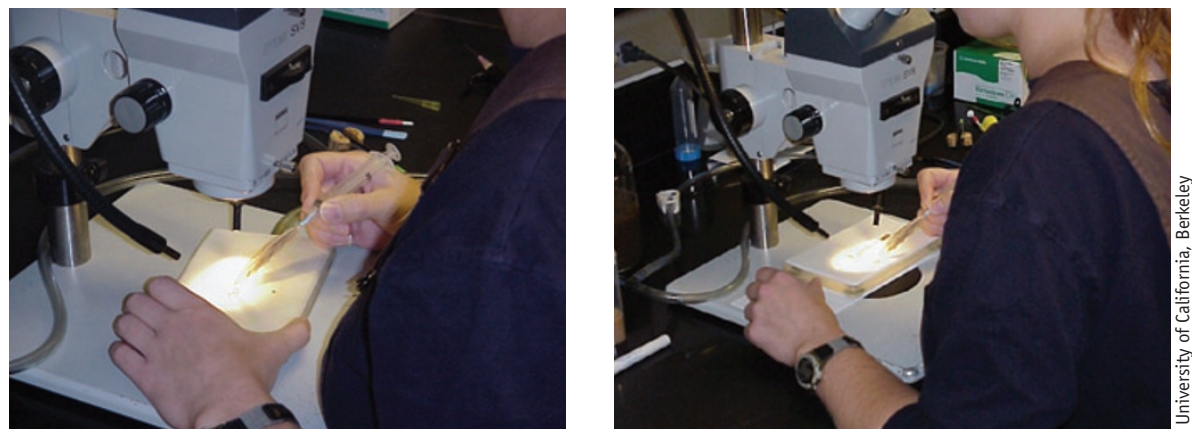

The fingers and thumb of the left hand are using an awkward and forceful posture to maneuver the $\mathrm{CO}_{2}$ pad in a fly lab (left). The left hand adopts a less stressful posture because the fingers and thumb grasp a knob that has been attached to manipulate the pad (right).

risks, not to mention the musculoskeletal damage induced by endless hours of pipetting. Yet not every hazard is declining. Now that nearly every work cubicle sports a computer, repetitive stress injuries actually are on the rise.

At the University of California, Berkeley, $40 \%$ of the injuries reported in the 74 labs in the molecular and cellular biology department were attributable to repetitive strain, according to Mark Freiberg, director of the university's Office of Environment, Health and Safety; the remaining $60 \%$ were divided evenly between slip-and-fall injuries, exposures to chemicals or ultraviolet light, and cuts from glassware and other objects.

"When we cover all the lab hazards, it's easy to show some really attention-getting photos of lab fires and burns, and that makes a real impression," Freiberg says. "It's hard to make an impression about chronic wrist pain, but that's not a trivial injury."
And robots, for all their convenience, pose potential risks too, says White. "People can get crush injuries," he says. "The robots come with safety devices, but sometimes scientists defeat safety devices [such as light sensors that are supposed to detect a user's hand] for reasons of convenience."

Though he has never seen such an event happen, White says it is a concern, especially at a facility like the Sanger Institute, which has an abundance of robots to feed its sequencing and genomics pipelines.

\section{Biosafety hazards}

Biological hazards also are evolving. Pathogenic organisms have been and continue to be particularly worrisome, not to mention a regulatory nightmare, especially in light of stricter policies put into place after the World Trade Center and anthrax attacks. A recent study estimated that red tape has increased the cost

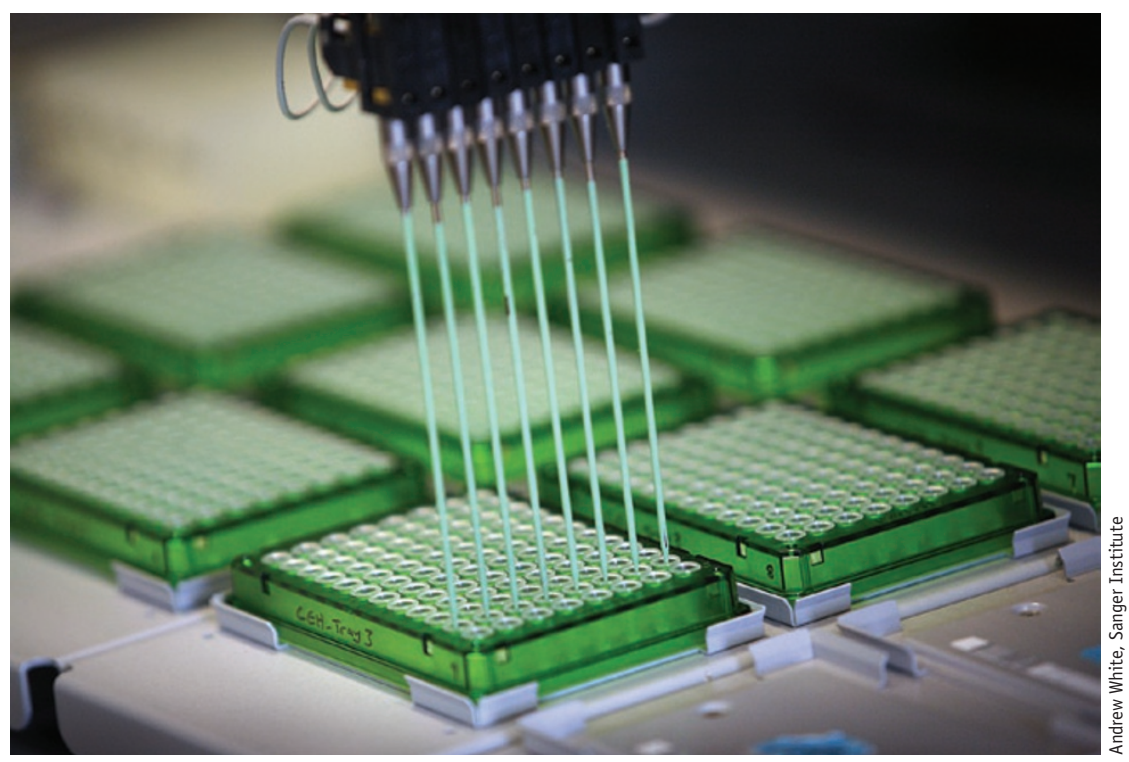

Laboratory robots usually have built-in safety devices, but researchers sometimes bypass them. 
of work on such "select agents" as Bacillus anthracis and Ebola virus from about $\$ 60,000$ per paper before rules were tightened to around $\$ 330,000$ today ${ }^{2}$.

But even non-select agents can raise safety flags. The Protein Chemistry Laboratory at SAIC-Frederick offers surface plasmon resonance and mass spectrometry services to the National Cancer Institute and the National Institutes of Health. John Simpson, senior scientist and the lab's acting safety officer, says the lab has had just two incidents in the past nine years: a cut resulting from broken glassware and a back injury caused by moving a refrigerator.

But it is the biological samples his team handles that gives Simpson pause. "We have no clue what the provenance of a sample is," he says. Given the lab's location-Fort Detrick, Maryland, USA, home of the US Army Medical Research Institute of Infectious Diseases, whose biosafety level 4 facility handles the worst of the worst, pathogenically speaking-“our awareness of safety is heightened, and we treat samples as such," Simpson says. He quips, "we treat everything as if it's Ebola with a pinch of HIV and go from there."

The same philosophy holds at the University of Wisconsin, Madison. "More of what we are seeing now is what we would label nonconventional blood-borne pathogen exposures," says occupational health officer Jim Morrison. A "nonconventional blood-borne pathogen," Morrison explains, includes everything from recombinant viruses to stem cells-anything that potentially poses a risk of infection.

Once the province of microbiologists who were trained to handle them, recombinant lentiviruses and adenoviruses can now be purchased as kits by any green graduate student with a purchase order. But without proper training in virology, these scientists may not recognize the potential danger such reagents hold: though such hazards have not yet been observed and are deemed unlikely, these viruses potentially can mix with undetected pathogens if introduced into cultured human cells to produce a replication-competent, infectious particle.

"It's an accessibility thing," Cook says. "Kits are really easy to get. You don't have to think about the viral life cycle and what can go wrong. It's just a black box."

Similarly, though researchers may not think of them that way, human stem cells also pose an infection risk. These cells typically are screened for known viruses, but unknown pathogens can nevertheless slip through. As a result, the standard practice at University of Wisconsin is to consider stem cells potential blood-borne pathogens and follow precautions developed for human blood, says Jim Turk, assistant director of environment, health and safety.

Researchers handling those cells and the resulting biological products must therefore use universal precautions-gloves, safety glasses and lab coats. And spills, aerosols, needle sticks and so on, can no longer be simply cleaned with bleach and ignored; they must be considered potential infections. If a researcher reports an incident and expresses a concern for personal safety, Morrison's office will refer that individual to an infectious disease specialist.

Currently medical personnel are illequipped to handle such situations, says Morrison. Occupational health providers are trained to deal with typical workplace issues: conventional blood-borne pathogens, musculoskeletal injuries, trauma from slips and falls, and so on. In the future, he predicts, large research institutions will be looking for occupational medical personnel who can assess situations involving more esoteric agents. "The challenge for the employers," he says, "is to work with the clinical providers to make sure they have a good understanding of [how to deal with] that."

To Turk's knowledge, no actual infections attributable to stem cell biology or recombinant materials have occurred at University of Wisconsin, Madison, which, as the birthplace of human embryonic stem cell research has a large and active stem cell research program. But, "[when] you get a lot of people handling stuff, accidents are going to happen," he says. "We tend to err on the side of caution."

1. Kemsley, J.N. Chem. Eng. News 87, 29-34 (2009).

2. Dias, M.B., Reyes-Gonzalez, L., Veloso, F.M. \& Casman, E.A. Proc. Natl. Acad. Sci. USA 107, 9556-9561 (2010).

Jeffrey M. Perkel is a freelance science writer based in Pocatello, Idaho, USA (jeff@jeffreyperkel.com).

\begin{tabular}{ll} 
Company & Web address \\
\hline $3 M$ & http://solutions.3m.com/ \\
Advnt Biotechnologies & http://www.advnt.org/ \\
Ansell Limited & http://www.ansell.com/ \\
\hline Assay Technology & http://www.assaytech.us/ \\
\hline ChemGlass Life Sciences & http://www.chemglass.com/ \\
Cole-Parmer & http://www.coleparmer.com/ \\
\hline Eagle Manufacturing Company & http://www.eagle-mfg.com/ \\
Encon Safety Products & http://www.enconsafety.com/ \\
Ergodyne & http://www.ergodyne.com/ \\
Fisher Scientific & http://www.fishersci.com/ \\
Glas-Col LLC & http://www.glascol.com/ \\
Guardian Equipment & http://www.gesafety.com/ \\
\hline Justrite Manufacturing & http://www.justritemfg.com/ \\
Lab Safety Supply & http://www.labsafety.com/ \\
Landau & http://www.landau.com/ \\
\hline
\end{tabular}

Company
Marvel Scientific
NPS Corp
PerkinElmer
Safety Emporium
Safety Glasses USA
Safety Supply America
Saf-T-Gard International
Scientific Materials Company
Seton Identification Products
Sigma Aldrich
Speakman Company
Supply Line Direct, NCH Corporation
Thermo Scientific
VWR

Web address

http://www.marvelscientific.com/ http://www.npscorp.com/spilfyter/

http://www.perkinelmer.com/ http://www.safetyemporium.com/ http://www.safetyglassesusa.com/ http://www.safetysupplyamerica.com/ http://www.saftgard.com/ http://www.scimatco.com/ http://www.seton.com/ http://www.sigmaaldrich.com/safetycenter.html

http://www.speakmancompany.com/ http://www.supplylinedirect.com/ http://www.thermoscientific.com/ http://vwrlabshop.com/ 